\title{
COMUNICACIÓN
}

\section{DIAGNÓSTICO ALIMENTICIO Y COMPOSICIÓN QUÍMICO NUTRICIONAL DE LOS PRINCIPALES INSUMOS DE USO PECUARIO DEL VALLE DEL MANTARO}

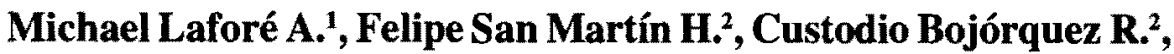 \\ Teresa Arbaiza $\mathrm{F}^{2}$ y Fernando Carcelén $\mathrm{C}^{2}{ }^{2}$
}

\section{Abstract}

\begin{abstract}
Dairy cattle breeders $(n=67)$ in the Mantaro valley (Junin, Peru) were surveyed in regard to feeding practices and the chemical-nutritional components of 68 feeds were analyzed for dry matter, raw protein, neutral detergent fiber, digestibility in vitro of dry matter, total digestible nutrients, metabolizable and digestible energy. Semintensive production situations predominate (78\%) and legume-like grasses $(94 \%)$, principally rye grass and clover $(81 \%)$, comprise the bulk of the diet. Grazing, supplemented with cut grass, grains and minerals given in the corrals, is the principal feeding strategy (77\%), and maize is the most frequently used harvest residue ( $81 \%)$. Nutritional values of cattle diet components from different parts of Mantaro valley are presented.

Key words: Feeding systems, dairy cattle, nutritional composition, digestibility.

Palabras clave: Sistemas de alimentación, ganado lechero, composición nutricional, digestibilidad.
\end{abstract}

La ganadería de la Sierra Central del Perú se basa principalmente en el uso de pasturas (naturales o cultivadas), rastrojos y sub productos agrícolas. Estos últimos usados intensamente en la época seca por escasez de pasturas.

Un factor importante para el eficiente uso del recurso alimenticio es el conocimiento de la composición química de las pasturas y demás alimentos; así como de los requerimientos nutricionales. En el Perú se han usado preferentemente las tablas de composición de alimentos y de requerimiento

\section{Práctica privada}

2 Laboratorio de Nutrición - FMV - UNMSM. E.Mail: d170032.unmsm.edu.pe animal de Morrison (1965) y del Consejo de Investigación de los Estados Unidos. Así mismo se han usado las tablas de composición para América Latina en sus versiones abreviada en español y completa en Inglés (Castro 1984).

Uno de los objetivos del presente estudio fue el definir los sistemas de crianza lechero predominantes en el Valle del Mantaro, en las provincias de Concepción y parte de Jauja. Estas provincias se hallan en el área de influencia de la Estación Experimental IVITA - El Mantaro, en la margen izquierda del Valle y cuentan con una gran concentración de productores. Toda el área se halla en la zona agroecológica baja (3000 - $3500 \mathrm{msnm}$ ). 
Para este objetivo se realizó una encuesta a 67 ganaderos. Las entrevistas se llevaron acabo en horas de la mañana.

La información recabada incluyó el tipo de crianza de sus animales, tipos de pastos y forrajes e insumos que utilizan en la alimentación de sus animales, utilización de subproductos de cosecha así como si dan o no suplemento.

Por otro lado, también se realizó la estimación de la composición química. Se tomaron muestras de los principales insumos alimenticios de uso pecuario en la zona: trigo, cebada, maíz amarillo, maíz corriente, harina de haba, afrecho de trigo, afrecho de cebada, afrecho de marigold, pasta de algodón, harina de pescado. Así mismo se tomaron muestras de forrajes tales como: alfalfa, avena, trébol rojo, trébol blanco, rye grass inglés, rye grass italiano, cebada, vicia, pasto ovillo, festuca alta, festuca media, Poa pratense, kikuyo (Pennisetum clandestinum), Medicago lupulina, Medicago ispida, chala de maíz, ensilaje de maíz, broza de arveja, broza de haba, broza de alcachofa, paja de cebada, paja de trigo, paja de avena.

Se realizaron análisis químicos proximales siguiendo el método goldfish para determinar extracto etéreo y el sistema Tecator para determinar proteína cruda; así mismo se determinó humedad, fibra cruda, extracto libre de nitrógeno y cenizas siguiendo los procedimientos descritos por la Association of Official Agricultural Chemestri (AOAC) (1990). Además se estimó la digestibilidad in vitro de la materia seca (DIVMS), y fibra detergente neutro (Goering y Van Soest, 1972). A partir de la digestibilidad in vitro de la materia seca (DIVMS) se estimó la energía digestible (4.4 x DIVMS/100) y la energía metabolizable (3.6 x DIVMS/ 100). El NDT se calculó a partir de la energía digestible estimada (ED en $\mathrm{Mcal} / \mathrm{kg}$ / 0.04409) (Nehring, 1973).

El sistema de crianza es predominantemente semiestabulado (78\%) (Cuadro 1). Los animales luego del ordeño son llevados a las áreas de pastoreo (08:00 a 09:00 horas) para regresar a sus corrales en las tardes (16:00 a 17:00 horas) y ser ordeñados nuevamente. La suplementación es una práctica muy difundida ( $88 \%$ ). Los suplementos más usados consisten en sales minerales y vitaminas comerciales y sal común. Algunos ganaderos preparan un concentrado casero a base de afrechillo y otros pocos dan concentrado comercial. Ambos concentrados se dan al momento del ordeño.

Cuadro 1. Sistemas de crianza y uso de suplementos en los establos lecheros encuestados.

\begin{tabular}{lcccccc}
\hline \multirow{2}{*}{ Sistema } & \multicolumn{2}{c}{ Con Suplementación } & \multicolumn{2}{c}{ Sin Suplementación } & \multicolumn{2}{c}{ Total } \\
\cline { 2 - 8 } & $\mathrm{n}$ & $\%$ & $\mathrm{n}$ & $\%$ & $\mathrm{n}$ & $\%$ \\
\hline Estabulado & 10 & 100 & -- & -- & 10 & 15.0 \\
Semi estabulado & 44 & 85 & 8 & 15 & 52 & 77.5 \\
Pastoreo & 5 & 100 & -- & -- & 5 & 7.5 \\
\hline Total & 59 & 88 & 8 & 12 & 67 & 100.0 \\
\hline
\end{tabular}

La composición nutricional de los principales insumos alimenticios usados en la alimentación del ganado se presenta en los Cuadros 2, 3, 4 y 5 . Así en el Cuadro 2 se presentan la composición nutricional de los granos. En general los resultados son coincidentes con lo reportado en la literatura. Sin embargo es notoria la alta calidad del grano de haba, principalmente por su alto contenido proteico. 
Cuadro 2. Composición química de granos.

\begin{tabular}{lccccccc}
\hline \multicolumn{1}{c}{ Granos } & $\begin{array}{c}\text { MS } \\
\%\end{array}$ & $\begin{array}{c}\text { PC } \\
\%\end{array}$ & $\begin{array}{c}\text { FDN } \\
\%\end{array}$ & $\begin{array}{c}\text { DIVMS } \\
\%\end{array}$ & $\begin{array}{c}\text { NDT } \\
\%\end{array}$ & $\begin{array}{c}\text { EM } \\
\mathrm{Mcal} / \mathrm{Kg}\end{array}$ & $\begin{array}{c}\mathrm{ED} \\
\mathrm{Mca} / \mathrm{Kg}\end{array}$ \\
\hline Avena & 93.12 & 11.04 & 32.97 & 85.32 & 84.92 & 3.07 & 3.74 \\
Cebada & 92.53 & 13.15 & 20.76 & 84.02 & 83.47 & 3.02 & 3.68 \\
Haba & 96.51 & 22.40 & 18.79 & 86.67 & 86.41 & 3.12 & 3.81 \\
& & & & & & & \\
Maíz & & & & & & & \\
Corriente & 91.95 & 5.83 & 14.74 & 79.46 & 79.16 & 2.86 & 3.49 \\
Amarillo & 92.83 & 7.52 & 13.64 & 80.38 & 79.84 & 2.89 & 3.52 \\
Trigo & 83.33 & 8.77 & 23.67 & 70.87 & 70.31 & 2.55 & 3.10 \\
\hline
\end{tabular}

MS = Materia seca, $\mathrm{PC}=$ Proteína cruda, FDN = Fibra detergente neutra, DIVMS = Digestibilidad in vitro de la materia seca, $\mathrm{NDT}=$ Nutrientes digestibles totales, $\mathrm{EM}=$ Energía metabolizable, $\mathrm{ED}=$ Energía digestible.

Cuadro 3. Composición química de residuos de cosecha.

\begin{tabular}{lccccccc}
\hline \multicolumn{1}{c}{ Residuos } & $\begin{array}{c}\text { MS } \\
\%\end{array}$ & $\begin{array}{c}\text { PC } \\
\%\end{array}$ & $\begin{array}{c}\text { FDN } \\
\%\end{array}$ & $\begin{array}{c}\text { DIVMS } \\
\%\end{array}$ & $\begin{array}{c}\text { NDT } \\
\%\end{array}$ & $\begin{array}{c}\text { EM } \\
\text { Mcal/Kg }\end{array}$ & $\begin{array}{c}\text { ED } \\
\text { Mcal/Kg }\end{array}$ \\
\hline $\begin{array}{l}\text { RASTROJOS } \\
\text { Alcachofa }\end{array}$ & 34.56 & 15.99 & 64.36 & 57.25 & 59.93 & 2.06 & 2.51 \\
Arveja & 60.00 & 8.91 & 59.04 & 43.77 & 43.87 & 1.58 & 1.93 \\
Haba & 74.00 & 18.99 & 62.81 & 84.20 & 84.02 & 2.03 & 3.70 \\
Maíz & $\bullet$ & & & & & & \\
Ensilado & & & & & & & \\
planta s/choclo & 36.37 & 8.83 & 68.99 & 56.82 & 56.70 & 2.05 & 2.50 \\
planta + choclo & 25.65 & 3.72 & 50.83 & 64.06 & 63.70 & 2.17 & 2.65 \\
PAJAS & 28.42 & 5.08 & 49.92 & 71.03 & 70.78 & 2.56 & 3.12 \\
- Avena & & & & & & & \\
- Cebada & 81.25 & 2.35 & 75.05 & 30.39 & 30.17 & 1.09 & 1.33 \\
- Trugo & 93.13 & 2.08 & 71.59 & 32.15 & 31.75 & 1.15 & 1.40 \\
\hline
\end{tabular}

MS = Materia seca, $\mathrm{PC}=$ Proteína cruda, $\mathrm{FDN}=$ Fibra detergente neutra, DIVMS = Digestibilidad in vitro de la materia seca, NDT $=$ Nutrientes digestibles totales, $\mathrm{EM}=$ Energía metabolizable, $\mathrm{ED}=$ Energía digestible. 
Cuadro 4. Composición química de residuos agroindustriales.

\begin{tabular}{lccccccc}
\hline \multicolumn{1}{c}{ Residuos } & $\begin{array}{c}\text { MS } \\
\%\end{array}$ & $\begin{array}{c}\text { PC } \\
\%\end{array}$ & $\begin{array}{c}\text { FDN } \\
\%\end{array}$ & $\begin{array}{c}\text { DIVMS } \\
\%\end{array}$ & $\begin{array}{c}\text { NDT } \\
\%\end{array}$ & $\begin{array}{c}\text { EM } \\
\text { Mcal/Kg }\end{array}$ & $\begin{array}{c}\text { ED } \\
\text { Mcal/Kg }\end{array}$ \\
\hline $\begin{array}{l}\text { Algodón } \\
\text { - pasta }\end{array}$ & 91.20 & 42.47 & 12.32 & 62.28 & 61.91 & 2.24 & 2.73 \\
$\begin{array}{l}\text { Cebada } \\
\text { - afrecho }\end{array}$ & 93.87 & 14.98 & 33.69 & 63.98 & 63.51 & 2.30 & 2.80 \\
- cáscara & 95.62 & 7.32 & 79.90 & 40.35 & 40.15 & 1.45 & 1.77 \\
$\begin{array}{l}\text { Marigold } \\
\text { - afrecho }\end{array}$ & 90.63 & 15.73 & 42.39 & 56.47 & 56.25 & 2.03 & 2.48 \\
$\begin{array}{l}\text { Pescado } \\
\text { - harina }\end{array}$ & 94.03 & 42.89 & - & 68.05 & 80.06 & 2.46 & 3.00 \\
$\begin{array}{l}\text { Trigo } \\
\text { - afrechillo }\end{array}$ & 93.10 & 17.29 & 50.23 & 60.28 & 60.10 & 2.17 & 2.65 \\
\hline
\end{tabular}

MS = Materia seca, PC=Proteína cruda, FDN=Fibra detergente neutra, DIVMS=Digestibilidad in vitro de la materia seca, $\mathrm{NDT}=$ Nutrientes digestibles totales, $\mathrm{EM}=$ Energía metabolizable, $\mathrm{ED}=$ Energía digestible.

Cuadro $5 . \quad$ Composición química de pastos y forrajes.

\begin{tabular}{lccccccc}
\hline \multicolumn{1}{c}{ PASTOS/ } & MS & PC & FDN & DIVMS & NDT & EM & ED \\
\multicolumn{1}{c}{ FORRAJES } & $\%$ & $\%$ & $\%$ & $\%$ & $\%$ & Mcal/Kg & Mcal/Kg \\
\hline Alfalfa & 37.97 & 22.7 & 40.41 & 56.67 & 56.39 & 1.98 & 2.49 \\
Avena & 38.50 & 10.42 & 34.14 & 59.63 & 59.42 & 2.15 & 2.62 \\
Festuca alta & 22.00 & 11.14 & 46.18 & 62.52 & 62.23 & 2.25 & 2.74 \\
Festuca media & 25.94 & 12.25 & 46.83 & 63.83 & 63.55 & 2.29 & 2.80 \\
Kikuyo & 27.64 & 24.19 & 49.95 & 52.40 & 52.17 & 1.89 & 2.30 \\
Medicago lupulina & 46.08 & 19.07 & 37.80 & 45.88 & 45.59 & 1.65 & 2.01 \\
Pasto ovillo & 26.68 & 13.58 & 46.24 & 64.63 & 64.56 & 2.33 & 2.68 \\
Poa pratensis & 28.96 & 16.32 & 43.64 & 63.63 & 63.28 & 2.29 & 2.79 \\
Rye grass ingles & 26.13 & 12.38 & 48.51 & 63.97 & 63.69 & 2.30 & 2.81 \\
Rye grass italiano & 25.40 & 9.60 & 51.00 & 57.19 & 55.50 & 2.06 & 2.51 \\
Trébol blanco & 31.80 & 27.03 & 54.36 & 58.88 & 58.63 & 2.12 & 2.68 \\
Trébol carretilla & 43.93 & 23.35 & 60.41 & 51.32 & 51.26 & 1.85 & 2.26 \\
Trébol rojo & 22.76 & 22.86 & 55.71 & 52.34 & 52.03 & 1.88 & 2.29 \\
Vicia +avena (70\%) & 26.90 & 31.23 & 49.73 & 45.73 & 45.59 & 1.65 & 2.01 \\
& 38.50 & 14.83 & 39.22 & 49.39 & 49.22 & 1.78 & 2.17
\end{tabular}

MS = Materia seca, $\mathrm{PC}=$ Proteína cruda, FDN = Fibra detergente neutra, DIVMS = Digestibilidad in vitro de la materia seca, NDT = Nutrientes digestibles totales, $\mathrm{EN}=$ Energía metabolizable, $\mathrm{ED}=$ Energía digestible. 
La composición nutricional de los residuos de cosecha se presenta en el Cuadro 3. De estos insumos resalta la alta calidad de la alcachofa, insumo que tiene un gran potencial de uso en la zona; toda vez que su cultivo es cada vez mayor debido a su alta demanda.

En el Cuadro 4 se presenta la composición nutricional de los residuos agroindustriales disponibles de la zona. En todos los casos la composición coincide con lo reportado en la literatura. Sin embargo es necesario señalar que la harina de pescado analizada es la disponible en el mercado local debiéndose recomendar tener cuidado en su compra debido a la gran variabilidad de su contenido proteico, debiéndose exigir un precio de acorde con dicho contenido.

Por último tenemos en el Cuadro 5 la composición nutricional de los principales pastos y forrajes de la zona. Estos resultados se deben tomar con precaución debido a que las muestras analizadas, tomadas en el momento de su utilización para la alimentación del ganado, varían dependiendo entre otros factores, de la edad de su utilización.

\section{Literatura Citada}

1. Association of Official Agricultural Chemisti. 1990. Official methods of analysis. 15 th ed. Washington, D. C, Association of Official Agricultural Chemists,

2. Castro, C. 1984. Tablas de composición de alimentos peruanos usados en el racionamiento animal. Tesis Bach. Med. Vet. UNMSM. Lima-Perú.

3. Goering, M. K. y P.J. Van Soest. 1972. Análisis de Fibra de forrajes (aparatos, reactivos, procedimientos y algunas aplicaiones). Agricultural Handbook $\mathrm{N}^{\circ}$ 379:15-16, Agricultural Research Service. United Estates Department of Agriculture.

4. Morrison, F.B. 1965. Alimentos y alimentación del ganado. Tomo II. 1ra. edición. Editorial Hispanoamericana. México.

5. Nehring, K. 1973. Feed evaluation and ration calculation based on net energy. J. An. Sci. 36:5. 